\title{
The Usefulness of Long Inflation Balloon Angioplasty Using a Perfusion Balloon for Intracoronary Thrombus
}

\author{
Shingo Watanabe ${ }^{*}$ and Michio Usui \\ The Department of Cardiology, Tokyo Yamate Medical Center, Tokyo, Japan

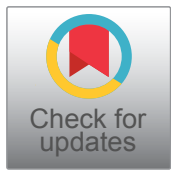

*Corresponding author: Shingo Watanabe, The Department of Cardiology, Tokyo Yamate Medical Center, Tokyo, Japan

\begin{abstract}
The beneficial effect of percutaneous coronary intervention $(\mathrm{PCI})$ in patients with acute coronary syndrome (ACS) has been well established. $\mathrm{PCI}$ for ACS, especially for thrombotic lesions, is difficult to treat due to distal embolization or residual thrombus. We experienced a case in which long balloon inflation was effective in PCI for ACS due to thrombotic lesions.

Here we introduce the case that long inflation balloon angioplasty using a perfusion balloon was useful in $\mathrm{PCl}$ for thrombus lesion.
\end{abstract}

\section{Keywords}

Long inflation balloon angioplasty, Perfusion balloon, Acute coronary syndrome, Thrombus lesion

\section{Text}

\section{Learning objective}

The beneficial effect of $\mathrm{PCl}$ in patients with ACS has been well established. However, distal embolization is sometimes observed during $\mathrm{PCl}$ for thrombus lesion. Thrombus aspiration therapy may be useful in $\mathrm{PCl}$ for thrombotic lesions. However, feasible alternative methods are limited when thrombus aspiration therapy failed. There is a need for treatment options to for thrombotic lesions for which thrombus aspiration therapy has failed. We introduce the case that long inflation balloon angioplasty using a perfusion balloon was useful in $\mathrm{PCl}$ for thrombus lesion.

\section{Introduction}

Intracoronary thrombus followed by a rupture of unstable vulnerable plaque is well known cause of ACS.
The beneficial effect of $\mathrm{PCl}$ in patients with ACS has been well established. However, distal embolization is sometimes observed during $\mathrm{PCl}$ for thrombus lesion [1]. No reflow phenomenon is caused by the distal embolization. No reflow phenomenon is closely related to the clinical outcome of patients, and is one of the leading causes of death after PCl [2].

Thrombus aspiration therapy and distal protection device may be useful in $\mathrm{PCl}$ for thrombotic lesions. In Japan, thrombus aspiration therapy is routinely performed for ACS due to thrombotic lesions. However, there is little evidence in previous studies that these treatments are useful $[3,4]$. We introduce the usefulness of long inflation balloon angioplasty using a perfusion balloon (Ryusei, Kaneka, Japan) for thrombus lesion to prevent the distal embolization.

\section{Case}

A 68-year-old male was admitted to our hospital with severe chest pain. Electrocardiography on arrival showed ST-segment elevation in leads II, III, and aVF. He was diagnosed with inferior ST-segment elevation myocardial infarction (STEMI).

Emergent coronary angiogram (CAG) was performed. CAG indicated total thrombotic occlusion at the proximal RCA and no organic stenosis in the left coronary arteries (Figure 1a). Primary $\mathrm{PCl}$ was performed under the diagnosis of inferior STEMI.

The 6F JR 4 (TAIGA, Medtronic, USA) was engaged to the RCA from the radial artery and guidewire (RunthroughNS, Terumo, Japan) was passed. Angiography showed thrombotic lesions. So, thrombectomy was at- 
(a)

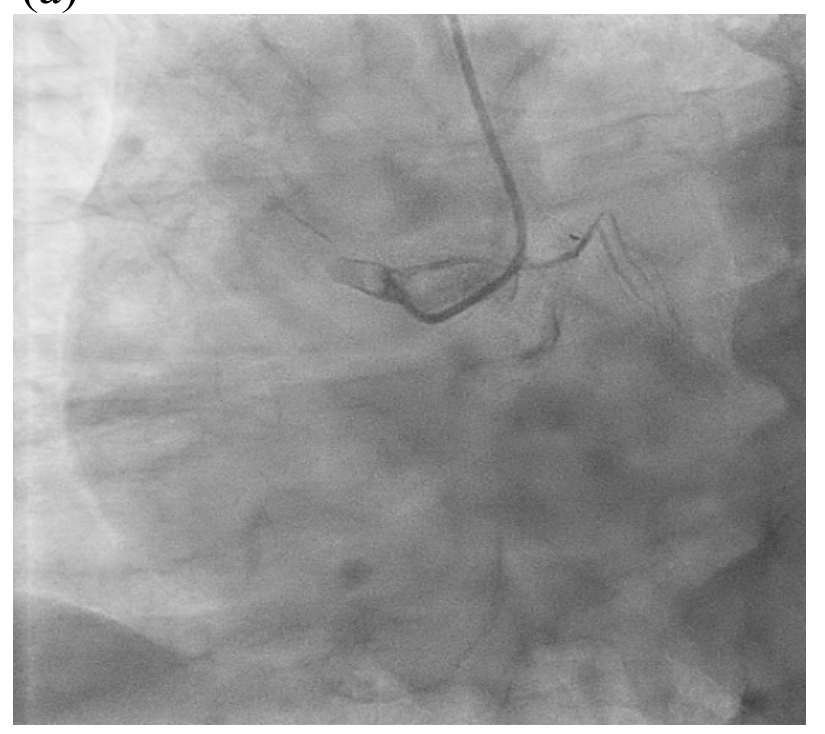

(c)

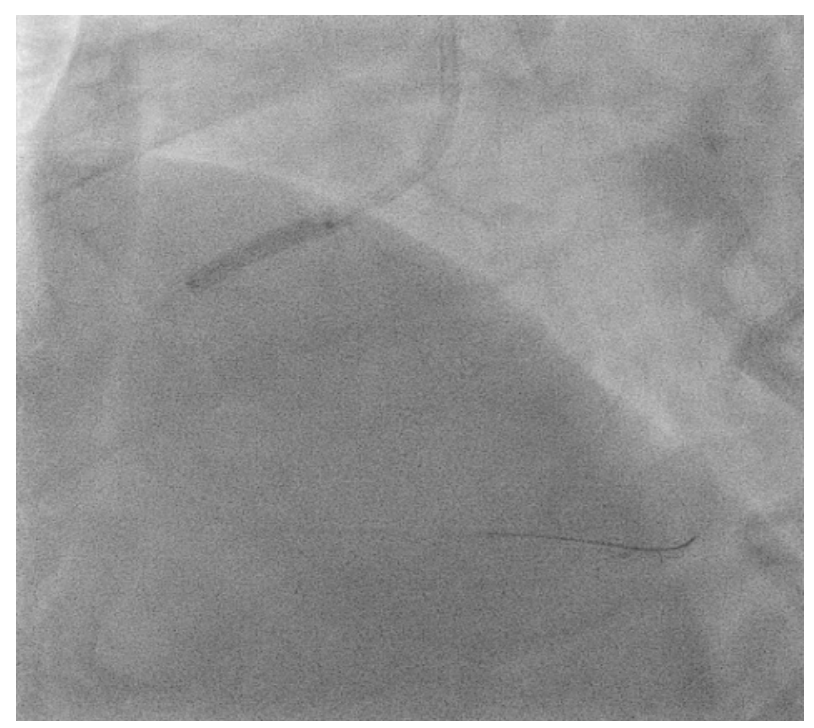

(e)

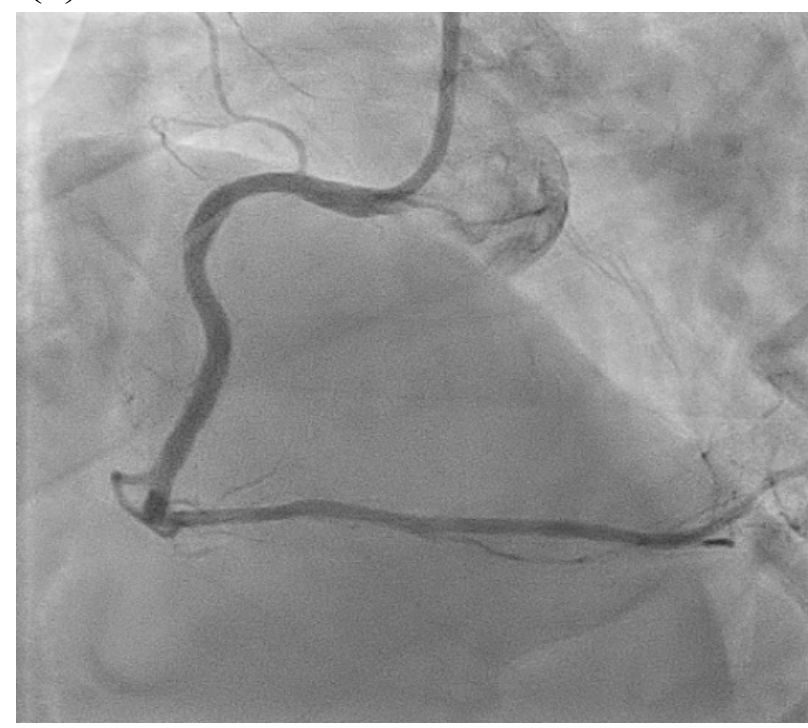

(b)

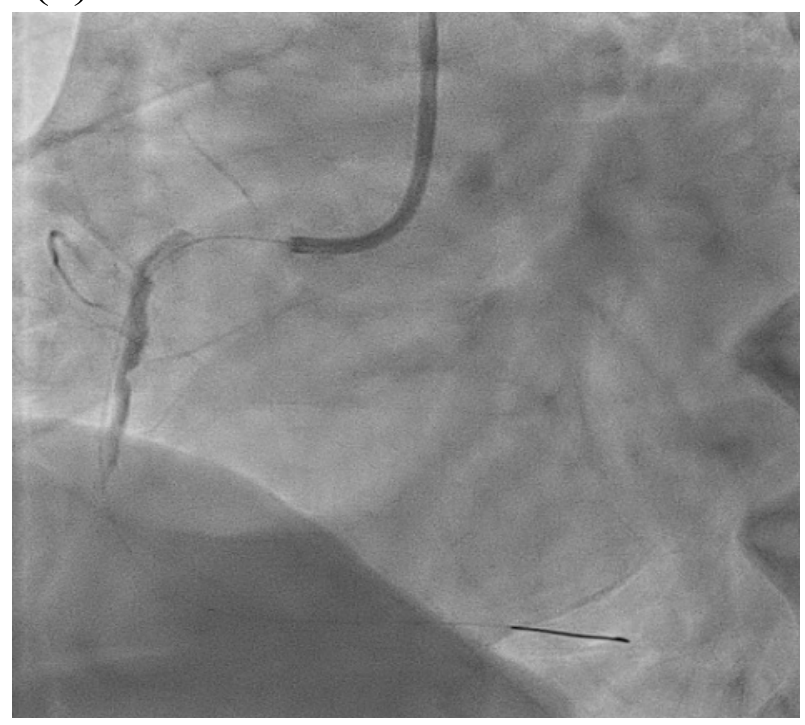

(d)

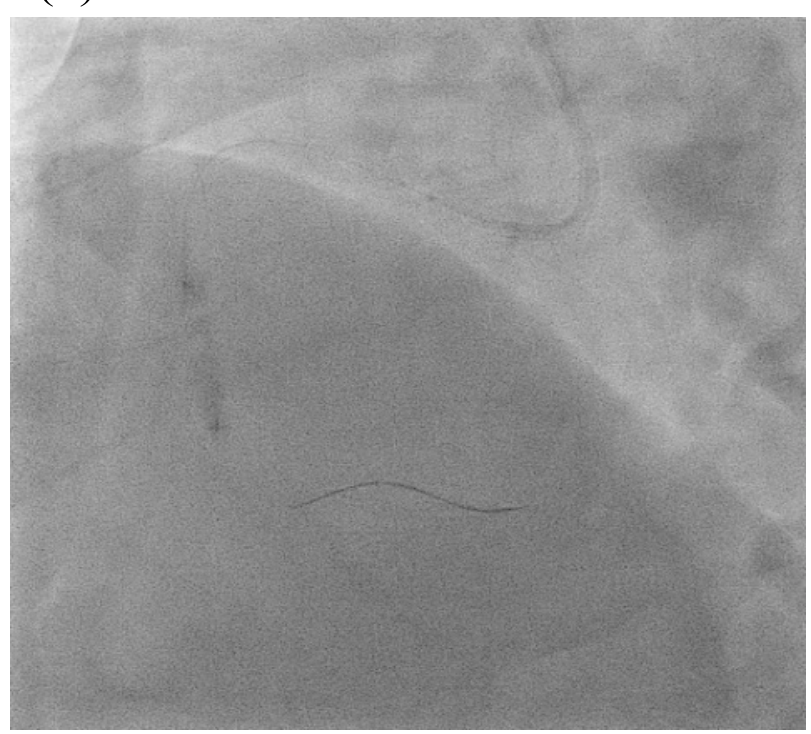

(f)

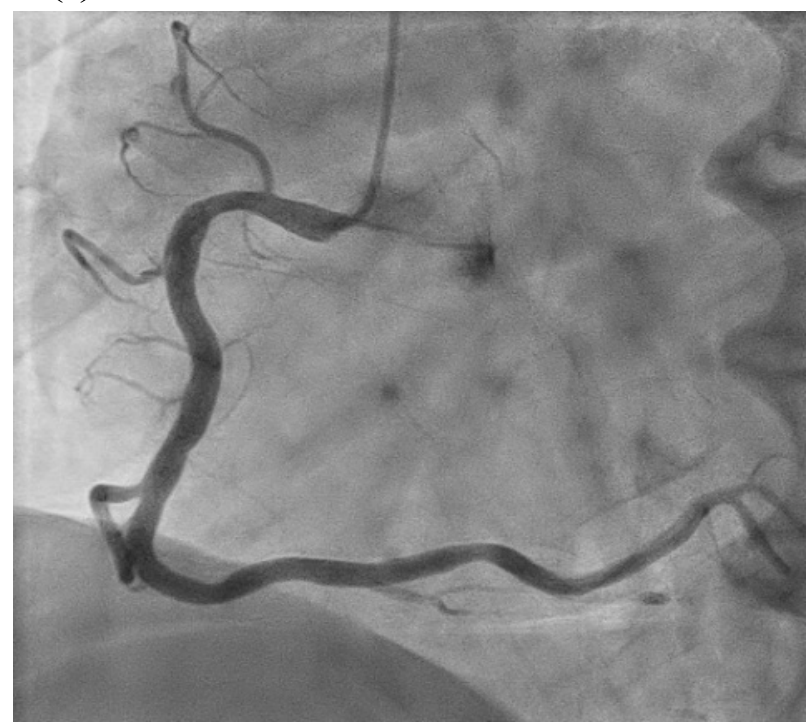

Figure 1: a) CAG indicated total thrombotic occlusion at the proximal RCA; b) CAG after thrombectomy indicated severe stenosis in the proximal RCA and occlusion in the middle RCA with massive thrombus; c\&d) Long inflation for 5 minutes each in the proximal and the mid RCA by the perfusion balloon; e) Final angiogram indicated no stenosis; f) Follw up CAG 8 days after initial $\mathrm{PCl}$. 
tempted several times using an aspiration catheter ( $\mathrm{Re}$ birth Pro2, Medtronic, USA). A red thrombus was aspirated from proximal $R C A$.

Subsequent angiogram indicated severe stenosis in the proximal RCA and occlusion in the middle RCA with massive thrombus (Figure $1 \mathrm{~b}$ ). It was considered that balloon expansion and stent placement for thrombotic lesions may cause no-reflow.

We performed long inflation for 5 minutes each in the proximal and the mid RCA by the perfusion balloon (Ryusei 3.5 mm Kaneka, Japan) (Figure 1c and Figure 1d).

Subsequent angiogram indicated an improvement in stenosis and good distal flow without slow flow (Figure $1 e)$. The procedure was completed without stenting.

In the early postoperative period, the ST elevation on the electrocardiogram also improved and the LV wall motion also improved.

Follow up CAG 8 days after initial $\mathrm{PCl}$ indicated no restenosis and TIMI flow Grade III (Figure 1f).

\section{Discussion}

Thrombotic lesions are difficult to treat due to distal emboli or residual thrombus. Distal embolization is a cause of no-reflow phenomenon. Thrombus aspiration therapy may be useful in $\mathrm{PCl}$ for thrombotic lesions. However, feasible alternative methods are limited when thrombus aspiration therapy failed.

Long inflation balloon angioplasty using a perfusion balloon was thought to be able to treat thrombotic lesions without causing distal emboli by holding the thrombus into the coronary artery wall (Figure 2). We performed long inflation using perfusion balloon for 6 cases of ACS lesions mainly caused by thrombus from January to December, 2019. In all 6 cases, CAG after long inflation indicated an improvement in stenosis and good distal flow without slow flow. All 6 cases were treated without stents. We thought that long balloon inflation technique is most effective for patients with massive thrombus and small plaque volume.

There are also some other treatment options for preventing distal embolization. Distal protection device is one of the treatment options to prevent distal embolization. In this case, Coronary flow after thrombectomy was TIMIO, so, it was difficult to use the distal protection device.

Some studies have shown that Glycoprotein IIb/IIla inhibitors reduce the incidence of no-reflow [5]. However, it cannot be used in Japan.

We set the inflation time of the long balloon inflation technique to 5 minutes. There is no scientific basis for setting the balloon inflation time to 5 minutes. In our case, we were able to perform $\mathrm{PCl}$ by long balloon inflation for 5 minutes without any significant thrombus remaining. More research is needed to determine the optimal extension time.

\section{Limitation}

This is a case series, and all procedures were performed by a single operator. Further research is needed.

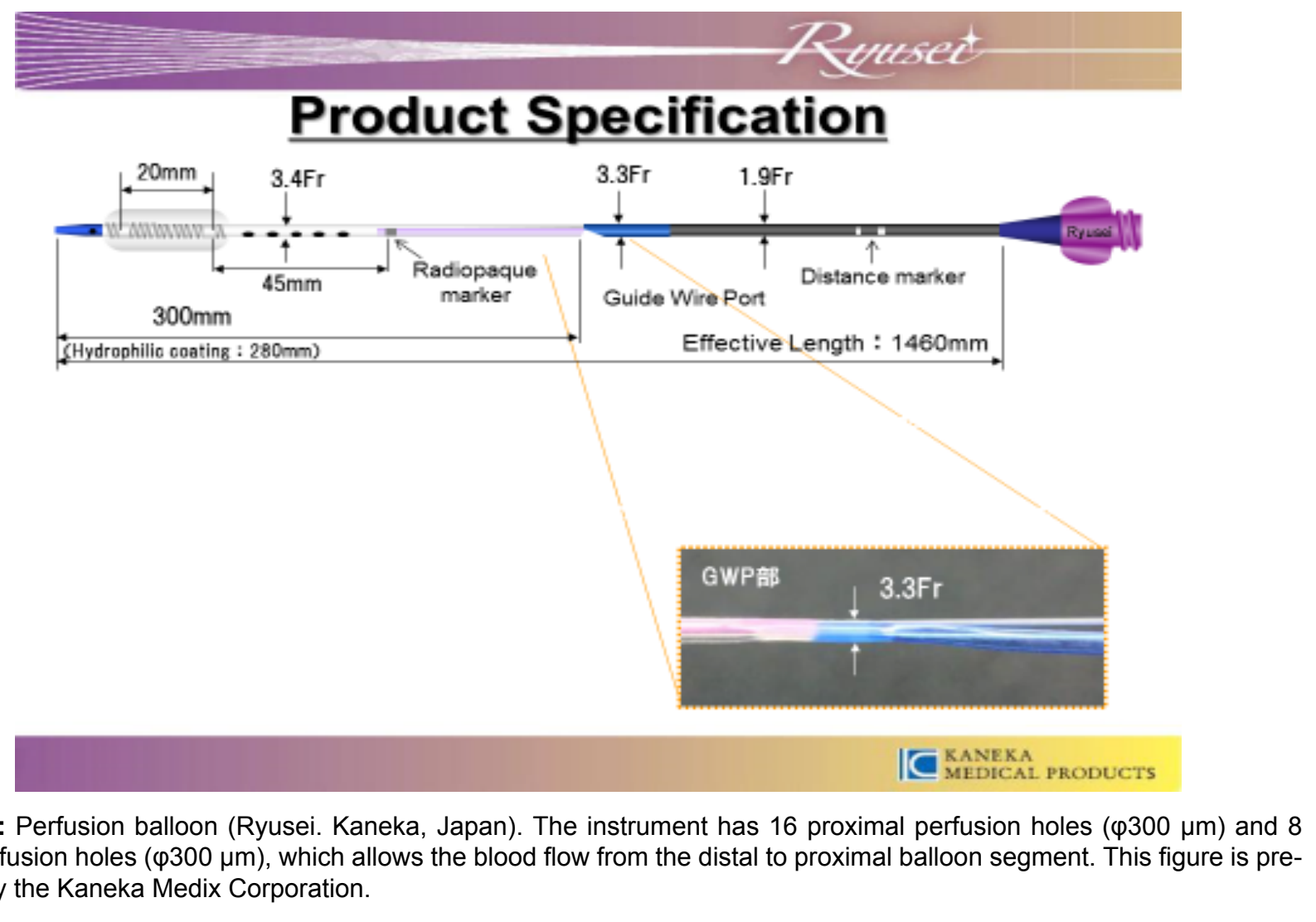




\section{Conclusion}

Long inflation balloon angioplasty using a perfusion balloon is a useful technique in thrombus lesion to prevent distal embolization.

\section{Conflict of Interest}

The authors declare that there is no conflict of interest.

\section{Notes}

The article conformed to the ethical guidelines of the 1975 Declaration of Helsinki.

The article protocol was approved by the local Institutional Committee on Human Research at our institution.

\section{Funding Information}

The sponsor had no control over the interpretation, writing, or publication of this work.

\section{References}

1. Niccoli G, Scalone G, Lerman A, Crea F (2016) Coronary microvascular obstruction in acute myocardial infarction. Eur Heart J 37: 1024-1033.

2. Harrison Robert W, Aggarwal Atul, Ou Fang-shu, Klein Lloyd W, Rumsfeld John S, et al. (2013) Incidence and outcomes of no-reflow phenomenon during percutaneous coronary intervention among patients with acute myocardial infarction. Am J Cardiol 111: 178-184.

3. De Luca G, Suryapranata H, Stone GW, Antoniucci D, Neumann FJ, et al. (2007) Adjunctive mechanical devices to prevent distal embolization in patients undergoing mechanical revascularization for acute myocardial infarction: A meta-analysis of randomized trials. Am Heart J 153: 343-353.

4. Lagerqvist B, Frobert O, Olivecrona GK, Gudnason T, Maeng M, et al. (2014) Outcomes 1 year after thrombus aspiration for myocardial infarction. N Engl J Med 371: 11111120.

5. Kurt Huber, David R Holmes Jr, Arnoud W. van 't Hof, Gilles Montalescot, Philip E Aylward, et al. (2010) Use of glycoprotein Ilb/llla inhibitors in primary percutaneous coronary intervention: Insights from the APEX-AMI trial. Eur Heart $\mathrm{J}$ 31: 1708-1716. 